\title{
Dynamics of Molecular Motors with Finite Processivity on Heterogeneous Tracks
}

\author{
Yariv Kafri*, David K. Lubensky\# and David R. Nelson* \\ * Department of Physics, Harvard University, Cambridge, MA 02138 and \\ \# BioMaPS Institute, Rutgers University, Piscataway NJ 08854 and Bell Labs, Lucent Technologies, Murray Hill, NJ 07974
}

(Dated: November 6, 2018)

\begin{abstract}
The dynamics of molecular motors which occasionally detach from a heterogeneous track like DNA or RNA is considered. Motivated by recent single molecule experiments, we study a simple model for a motor moving along a disordered track using chemical energy while an external force opposes its motion. The motors also have finite processivity, i.e., they can leave the track with a position dependent rate. We show that the response of the system to disorder in the hopping off rate depends on the value of the external force. For most values of the external force, strong disorder causes the motors which survive for long times on the track to be localized at preferred positions. However, near the stall force, localization occurs for any amount of disorder. Existence of localized states near the top of the band implies a stretched exponential contribution to the decay of the survival probability. To obtain these results, we study the complex eigenvalue spectrum of the time evolution operator. A similar spectral analysis also provides a very efficient method for studying the dynamics of motors with infinite processivity.
\end{abstract}

\section{INTRODUCTION}

Single molecule experiments which study molecular motors provide a powerful tool for understanding their function 1]. By applying a mechanical force, one can often discern details of their reaction steps. For example, a recent paper considered the motion of a kinesin moving along a microtubule under the influence of a force opposing its motion 2]. By measuring the relationship between the applied force, $F$, and the velocity of the kinesin, $v$, at different ATP concentrations it was possible to infer that the chemical reaction cycle contains at least one load dependent transition.

More recently, it has been possible to study, using similar techniques, the motion of RNA polymerase (RNAp) [3, 4], DNA polymerase (DNAp) [5], helicases [6] and $\lambda$-exonuclease [7]. In contrast to kinesin and myosin, which move along homogeneous polymer filaments, these motors move along DNA, which is inherently a heterogeneous track, with the energy landscape determined by the nucleotide sequence [8]. Indeed, the dynamics of these motors seems far richer than that of kinesin 2] or, say, myosin V [9], which move along periodic filaments. Theoretically, it is expected that heterogeneous and homogeneous (or periodic) tracks can give rise to very different motor dynamics. On long time and large length scales the motion of motors moving along a homogeneous track is described well by a random walker moving along a tilted potential. In contrast, the motion of molecular motors which use chemical energy to move along disordered filaments is described by a random walker moving on a random forcing energy landscape [10, 11]. The effective energy difference between two points separated by $m$ nucleotides scales as $\sqrt{m}$. The large energy barriers implied by such landscapes lead to anomalous dynamics [12] when the overall tilt of the energy landscape is small; the displacement of the motor grows as a sublinear power of time. For molecular motors this corresponds to applying an external force strong enough to place the system near the stall point of the motor.

The theories described above do not treat the effect on the dynamics of a (position-dependent) detachment probability of motors from the track. We will refer to models where no unbinding of the motor from the track is allowed as "infinite processivity" models. In this paper we study theoretically the effect of a finite processivity on the behavior of molecular motors. Previous work has considered the effect of detachment mainly on the dynamics of many molecular motors or with homogeneous tracks 13, 14, 15, 16, 17]. We consider a motor which can leave the polymer on which it is moving but can never rebind to it. This case is relevant to single molecule experiments which follow a specific motor or a dilute concentration of motors on a long DNA track. In such experiments (especially if a background hydrodynamic flow washes away detached motors) the probability of rebinding to the track after detachment is negligible.

We show that when molecular motors are moving along a homogeneous (or periodic) filament the long time dynamics of those motors that remain attached is unaltered by detachment events. However, for motors moving along a heterogeneous track this is not the case. For proteins such as RNAp and DNAp that walk along DNA, the detachment rate depends on the monomer on which the motor is located. The disorder in the detachment rates is thus correlated with the disorder in the hopping rates as both are determined by the same DNA sequence. When the detachment rate varies from monomer to monomer, the motors' dynamics can be influenced by spatially localized eigenfunctions of the evolution operator. For the regime where the displacement of the motor is linear as a function of time, strong disorder causes the last motors which remain attached to the track to stall out before falling off. Moreover, when the displacement of the motor as a function of time is sublinear, any amount of disorder in the hopping off rates causes the last motors which remain attached to the track to halt before falling off. A schematic representation of the resulting "phase diagram" for the dynamics is shown in Fig. प 


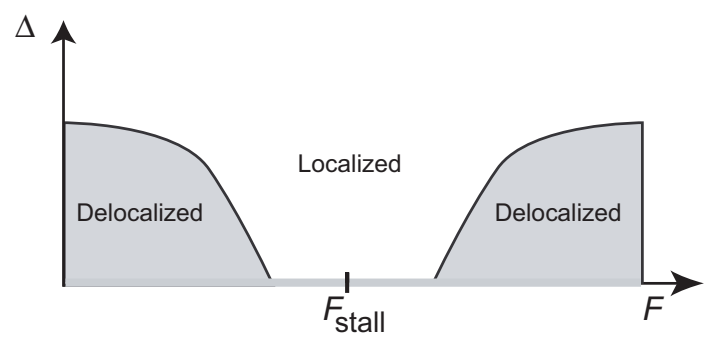

FIG. 1: Schematic behavior of the influence of disorder in the rates for leaving the track on the dynamics of the motors. $\Delta$ represents the strength of the disorder, measured through the variance of the hopping off rates divided by the square of their mean. Note that when $\Delta=0$ the motors are always delocalized except at $F_{\text {stall }}$. This is emphasized by the shading. It should be stressed that the notions "localized" and "delocalized" here refer to motors which remain on the track for long times.

To show these results we use methods developed in [18, 19], in the context of the physics of vortices, and numerically study the eigenvalue spectrum of the non-hermitian evolution operator for the probability distribution of the motor. As will be shown, the characteristics of this operator's spectrum (which include localized and delocalized states as well as a mobility edge) allow the long time, large length-scale dynamics to be obtained in a straightforward manner. Interestingly, the dynamics of motors on heterogeneous tracks with finite processivity is similar to the non-hermitian statistical mechanics arising in models of vortex physics [18, 19.

To support these results further we also study analytically a toy model which consists of a directed walker among traps with a broad distribution of release times. The model was studied for the case of infinite processivity in [12]. We show that in the infinite processivity case the model yields the same spectrum as observed in the numerics. Moreover, we show that for a motor with finite processivity, when the corresponding infinite processivity model shows sublinear drift, any amount of disorder in the hopping off rates leads to localization of the eigenfunction, consistent with our numerical results.

The paper is organized as follows: In Section II the model we study is introduced and known results for the infinite processivity limit are reviewed. In Section III the infinite processivity model is analyzed using the eigenvalue spectrum of the evolution operator. It is shown that the long time, large length-scale behavior of the model can be extracted from the properties of the spectrum. In Section IV we study the spectral properties of an evolution operator that takes account of detachment of motors from the track and their physical implications. Specifically, the implications for measurements of the probability of finding a motor on the track as a function of time are discussed. Finally, in Section V the analysis of the toy model is presented.

\section{THE MODEL}

The model we consider was introduced and studied in the limit of infinite processivity in [11. It is inspired by previous models of molecular motors $10,20,21,22,23$, but is simple enough so that exact solutions can be found, in the limit of infinite processivity, with and without disorder. The model is defined on a discrete lattice, $x=0,1,2 \ldots$, with distinct $a$ (even) and $b$ (odd) sites and a distance $a_{0}$ between lattice points. A monomer of the track (a nucleotide, say) is taken to be of size $2 a_{0}$. The arrangement is shown schematically in Figs. 2 and 3 . To model the two internal states of the model we take even sites to have an energy $\varepsilon=0$ while odd sites have an energy $\varepsilon=\Delta \varepsilon$. The transition rates depicted in Fig. 3 take the form

$$
\begin{aligned}
& w_{a}^{\vec{a}}=\left(\alpha e^{\Delta \mu / T}+\omega\right) e^{-\Delta \varepsilon / T-f / 2 T} \\
& w_{b}^{\leftarrow}=(\alpha+\omega) e^{f / 2 T} \\
& w_{a}^{\leftarrow}=\left(\alpha^{\prime} e^{\Delta \mu / T}+\omega^{\prime}\right) e^{-\Delta \varepsilon / T+f / 2 T} \\
& w_{b}^{\rightarrow}=\left(\alpha^{\prime}+\omega^{\prime}\right) e^{-f / 2 T}
\end{aligned}
$$

where we have set the Boltzmann constant to be $k_{B}=1$. Note that there are two parallel channels for the transitions. The first, represented by contributions containing $\alpha$ and $\alpha^{\prime}$, arise from utilization of the chemical energy $\Delta \mu$. The chemical energy difference could be, for example, a result of an excess concentration of NTP's (or just ATP) with respect to its thermal equilibrium value. The second channel, represented by the terms containing $\omega$ and $\omega^{\prime}$, correspond to thermal transitions unassisted by chemical energy. In addition, the externally applied force $F$, with $f=F a_{0}$, biases the motion. To model finite processivity we add rates $w_{\mathrm{off}}^{a}$ and $w_{\mathrm{off}}^{b}$ corresponding to the detachment from the track at even and odd sites respectively. 


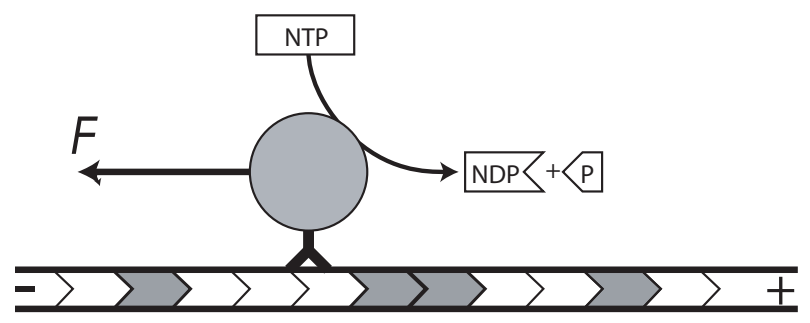

FIG. 2: Setup modelled. The motor is moving from the - end to the + end, driven by hydrolysis of nucleotide tri-phosphate. A force is pulling on the motor in the opposite direction. The track shown is made up of two types of monomers (depicted as shaded and white areas). Although this schematic suggests a microtubule with disorder in the protein constituents, we actually have in mind motors moving on DNA or RNA templates, with four distinct nucleotides. Elaborations which make our model more realistic at a microscopic level should not affect the predictions for long time, large length-scale dynamics.

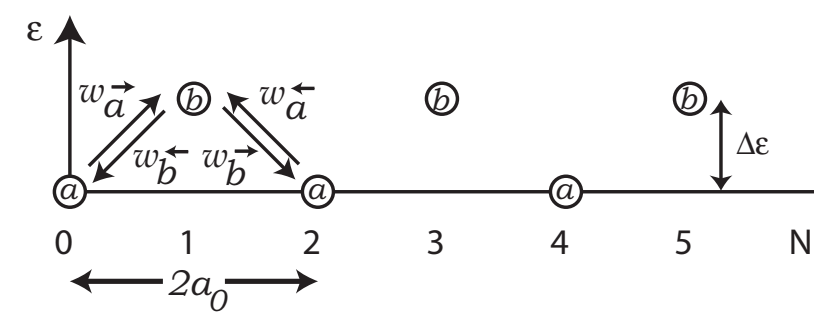

FIG. 3: Graphical representation of the model for molecular motors. The distinct even and odd sites are denoted by $a$ and $b$ respectively.

Before turning to the study of finite processivity we review some of the results which are known for the infinite processivity limit [1]. We show later that these results may be reproduced by studying the spectral properties of the evolution operator of the probability distribution. It is the spectral method that will allow us to determine the dynamics most readily when the processivity is finite. In the homogeneous case $\left(\alpha, \alpha^{\prime}, \omega\right.$ and $\omega^{\prime}$ independent of position), when no disorder is present the model is described on long times and large length-scales by a random walker moving along a potential with an overall tilt between two even sites which is given by

$$
\begin{aligned}
\Delta E & =T \ln \left(\frac{w_{a}^{\leftarrow} w_{b}^{\leftarrow}}{w_{a}^{\rightarrow} w_{b}^{\rightarrow}}\right) \\
& =T \ln \left(\frac{(\alpha+\omega)\left(\alpha^{\prime} e^{\Delta \mu / T}+\omega^{\prime}\right)}{\left(\alpha e^{\Delta \mu / T}+\omega\right)\left(\alpha^{\prime}+\omega^{\prime}\right)}\right)+2 f .
\end{aligned}
$$

We refer to such an energy landscape as an effective energy landscape. It is an alternative description of the dynamics associated with the rates of Eq. 10 which in general will not satisfy detailed balance since they describe nonequilibrium processes. Note that when $f=0$ and the chemical potential difference $\Delta \mu=0$, one has $\Delta E=0$ and no net motion is generated. Also, when there is directional symmetry in the transition rates $\alpha=\alpha^{\prime}, \omega=\omega^{\prime}$ (reflecting directional symmetry in the DNA track) and $f=0$ one has $\Delta E=0$ even when $\Delta \mu \neq 0$. Absent this symmetry, chemical energy can be converted to motion and an effective tilted potential is generated. Similar conditions for biased motion have been shown to exist for continuum models 22,23 . The effect of the externally applied force is simply to change the overall tilt in the potential. Thus, for motors moving along a homogeneous (or periodic) polymer, one expects the velocity to change continuously as $f$ is changed (see Fig. (4).

For motors moving on heterogeneous filaments the situation is very different. Here the set of parameters $\{p\}=$ $\left\{\alpha, \alpha^{\prime}, \omega, \omega^{\prime}, \Delta \varepsilon\right\}$ is drawn from a random distribution. Each set of parameters describes the dynamics on a given type of monomer. Using the results presented above it is easy to see using equation (2) that the total effective energy change after $m$ monomers is given by

$$
E(m)=\sum_{l=1}^{m} \Delta E(l) .
$$

Here, each $\Delta E(m)$ corresponds to an independent set of values of $\{p\}$ drawn randomly for the $\mathrm{m}^{\text {th }}$ monomer. Assuming 
that $\Delta E(m)$ is drawn from a random distribution with a finite variance, the effective energy landscape corresponds to a biased random walk. Such energy landscapes are typically referred to as random forcing energy landscapes.

The above scenario applies as long as the chemical potential difference $\Delta \mu \neq 0$. In the case when $\Delta \mu=0$ it is easy to see that $E(m)=0$ unless we allow for the energy at even sites also to vary and take the value $\varepsilon(m)$ [24]. In this case we obtain

$$
E(m)=2 f m+\varepsilon(m)
$$

corresponding to a random energy landscape provided $\varepsilon(m)$ has only short range correlations.

The dynamical behaviors of random walkers in random forcing or random energy landscapes have been studied in detail [12, 25]. Using the results of Derrida [25] one can calculate the transition points between the different regimes including the effect of randomness for our model [11]. Upon denoting averages over the disorder rates by an overbar one finds the following regimes.

Regime I: Ordinary biased diffusion occurs when

$$
f<-\frac{T}{4} \ln {\overline{\left(\frac{w_{a}^{\leftarrow} w_{b}^{\leftarrow}}{w_{a}^{\rightarrow} w_{b}^{\rightarrow}}\right)^{2}}}_{f=0}
$$

or

$$
f>\frac{T}{4} \ln {\overline{\left(\frac{w_{a}^{\rightarrow} w_{b}^{\rightarrow}}{w_{a}^{\leftarrow} w_{b}^{\leftarrow}}\right)^{2}}}_{f=0}
$$

where the subscript $f=0$ denotes that $f$ has been set to zero when evaluating the average. In this regime $\langle x\rangle=v t$ and $\left\langle x^{2}\right\rangle-\langle x\rangle^{2}=2 D t$ for long times, where the angular brackets denote an average over different thermal histories of a particle starting from a particular point. Another way of stating this result is that for large times the calculated velocity and diffusion constant, defined by the above relations, do not depend on the size of the time window, $t_{W}$, over which they are evaluated. This results only holds for biases satisfying Eqs. (5) and (6).

Regime II: The calculated diffusion constant now depends on the size of the time window, $t_{W}$, over which it is evaluated. The velocity does not. Namely, in this region $\langle x\rangle=v t$ and $\left\langle x^{2}\right\rangle-\langle x\rangle^{2} \sim t^{2 / \mu}$, where $1<\mu(f)<2$. In the infinite $t_{W}$ limit the diffusion constant, $D \equiv \lim _{t_{W} \rightarrow \infty}\left(\left\langle x\left(t_{W}\right)^{2}\right\rangle-\left\langle x\left(t_{W}\right)\right\rangle^{2}\right) / t_{W}$, diverges. This anomaly occurs in the ranges

$$
-\frac{T}{4} \ln {\overline{\left(\frac{w_{a}^{\leftarrow} w_{b}^{\leftarrow}}{w_{a}^{\rightarrow} w_{b}^{\rightarrow}}\right)^{2}}}_{f=0}<f \leq-\frac{T}{2} \ln {\overline{\left(\frac{w_{a}^{\leftarrow} w_{b}^{\leftarrow}}{w_{a}^{\rightarrow} w_{b}^{\rightarrow}}\right)_{f=0}}}
$$

and

$$
\frac{T}{2} \ln \overline{\left(\frac{w_{a}^{\rightarrow} w_{b}^{\rightarrow}}{w_{a}^{\leftarrow} w_{b}^{\leftarrow}}\right)} \quad \leq f<\frac{T}{4} \ln \overline{\left(\frac{w_{a}^{\rightarrow} w_{b}^{\rightarrow}}{w_{a}^{\leftarrow} w_{b}^{\leftarrow}}\right)^{2}}
$$

Regime III: Here both the velocity and the diffusion constant are functions of the size of the time window $t_{W}$, and $\langle x\rangle \sim t^{\mu}$ and $\left\langle x^{2}\right\rangle-\langle x\rangle^{2} \sim t^{2 \mu}$, where $\mu(f)<1$. In the infinite $t_{W}$ limit the velocity $v \equiv \lim _{t_{W} \rightarrow \infty}\left(\left\langle x\left(t_{W}\right)-x(0)\right\rangle\right) / t_{W}$ vanishes. The diffusion constant, $D \equiv \lim _{t_{W} \rightarrow \infty}\left(\left\langle x\left(t_{W}\right)^{2}\right\rangle-\left\langle x\left(t_{W}\right)\right\rangle^{2}\right) / t_{W}$, either vanishes or diverges depending on whether $\mu<1 / 2$ or $\mu>1 / 2$. This behavior occurs when

$$
\left.-\frac{T}{2} \ln \overline{\left(\frac{w_{a}^{\leftarrow} w_{b}^{\leftarrow}}{w_{a}^{\rightarrow} w_{b}^{\hookrightarrow}}\right)}\right)_{f=0} \leq f \leq \frac{T}{2} \ln \overline{\left(\frac{w_{a}^{\rightarrow} w_{b}^{\rightarrow}}{w_{a}^{\leftarrow} w_{b}^{\leftarrow}}\right)_{f=0}}
$$

Sinai diffusion: Exactly at the stall force $f_{s},\langle x\rangle=0$ and $\left\langle x^{2}\right\rangle \sim(\ln (t / \tau))^{4}$, where $\tau$ is the microscopic time needed to move across one monomer. The "stall force" corresponding to a disordered track is defined by

$$
f_{s}=\frac{T}{2} \overline{\ln \left(\frac{w_{a}^{\rightarrow} w_{b}^{\rightarrow}}{w_{a}^{\leftarrow} w_{b}^{\leftarrow}}\right)} \text { f=0 }
$$

The resulting behavior as the force is varied is summarized qualitatively in Fig. 廿 in the limit of an infinite averaging time window $t_{W}$. Most notable is the region of forces (region III) over which the displacement of the motor is sublinear and the usual long time velocity vanishes. Experiments are performed with finite $t_{W}$. The measured velocity then 


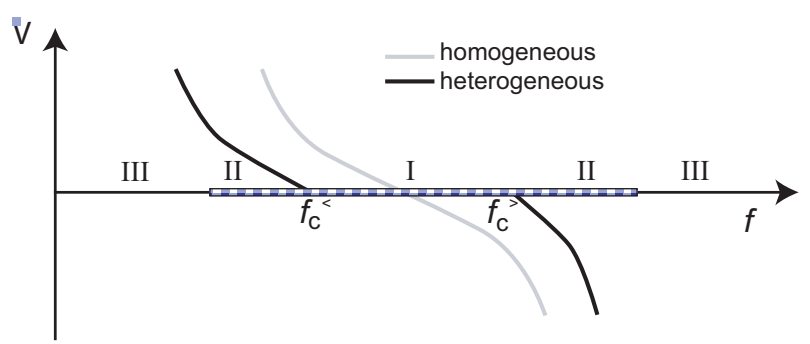

FIG. 4: Schematic behavior of the velocity for a heterogeneous linear motor track as a function of the applied force. It is assumed that chemical forces (from the NTP hydrolysis) lead to a positive velocity in the absence of a force. The anomalous dynamics arises in the vicinity of a stall force defined by Eq. 10] The different dynamical regimes defined in the text are denoted in the figure. The striped line on the $f$-axis denotes the region where anomalous diffusion is present.

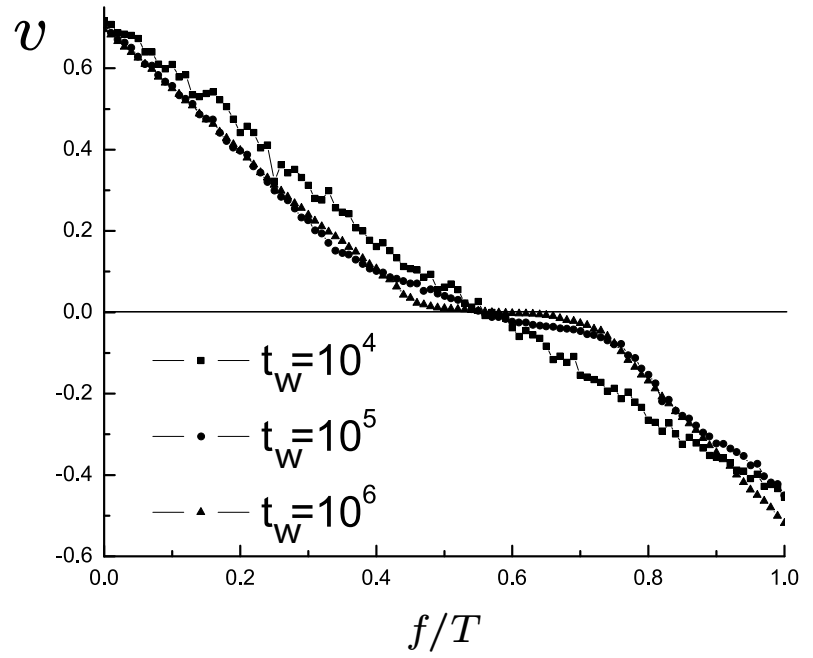

FIG. 5: The velocity as a function of $f / T$ for different values of $t_{W}$. Here $\Delta \mu / T=3$ and parameters were chosen with equal probability to be either $\{p\}=\{5,1,0.3,1,0\}$ or $\{p\}=\{4,0.1,0.7,1,0\}$ (see text for notation). The calculated regime of anomalous velocity is $0.5116<f / T<0.699$. Data incorporates 100 runs (thus averaging over thermal fluctuations) for a single realization of the disorder.

behaves in region III as $t_{W}^{\mu-1}$, smoothing the curve shown in Fig. 4 and naturally giving rise to a convex shape of the velocity-force curve. This convexity is demonstrated in Fig. [ 5 where the model was simulated and the velocity measured using different averaging time windows $t_{W}$ (see Appendix A for details). As is evident in this figure, the

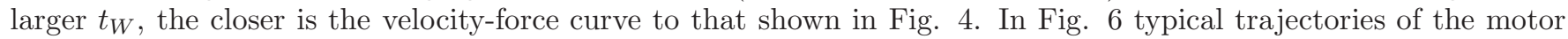
on the track are shown for different values of $f / T$ for a single realization of the disorder. Plateaus and jumps appear as one moves closer to the region of anomalous displacement, and the dynamics is controlled by deep minima in the effective energy landscape with rapid transitions between them. Such motion is typical of random forcing energy landscapes [12].

\section{INFINITE PROCESSIVITY: A SPECTRAL ANALYSIS APPROACH}

The behavior of the infinite processivity limit, as summarized above, is rather well understood. In this section we show that the dynamics with infinite processivity may also be deduced by an alternative approach: the spectral properties of the evolution operator of the probability density are exploited to deduce the long time, large length-scale properties of the model. The important features of the eigenvalues and eigenfunctions which characterize the long time dynamics are expected to be insensitive to details of the model. The method has previously been applied in the study of the physics of vortex lines in superconductors [18, 19] and population dynamics [26, 27]. Earlier studies considered the spectral properties of a random walker on a random forcing energy landscape after "gauging away" the external bias, making the evolution operator hermitian [12]. However, as we will show, a more direct approach reveals important features (such as complex eigenvalue spectra) which are not easily observed in the approach of [12]. 

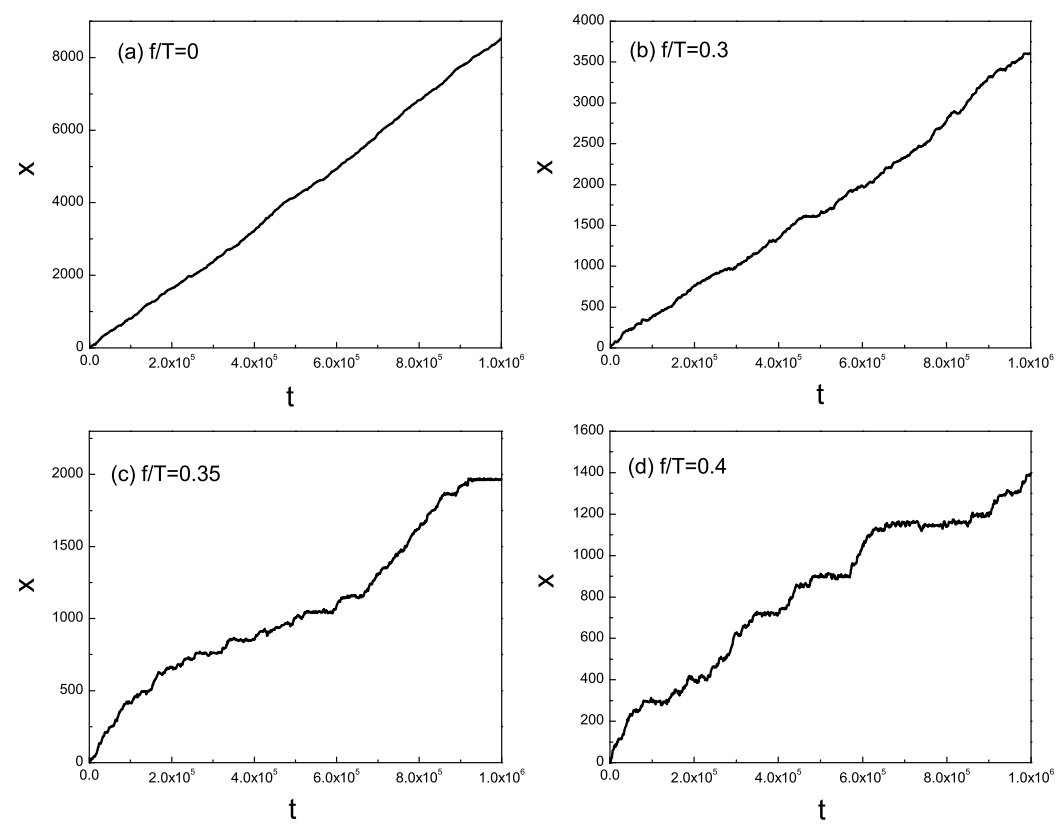

FIG. 6: Typical motor trajectories shown for the same parameters as Fig. 5 The values of $f / T$ are indicated in the figure. Note that the plateaus and jumps become more pronounced as $f$ increases toward the stall force $f_{s} \simeq 0.56$

Although here the approach is used to reproduce known results, it might prove useful in the study of more complicated models with an infinite processivity where an analytic solution is not possible. In addition, the spectral approach to the infinite processivity model will be very useful as a benchmark once we address the question of finite processivity.

In what follows, we will often consider a coarse-grained effective dynamics in which each new lattice site represents a unit cell, containing one $a$ and one $b$ site, of the original lattice (Eq. 1 and Fig. 3). The lattice spacing in this new coarse-grained model is $a=2 a_{0}$. Below we first review the spectrum (and the associate dynamics) of the trivial homogeneous model before turning to the disordered one.

\section{A. Homogeneous Model}

As discussed above the homogeneous problem is well described on long time-scales and large length-scales by a random walker moving along a tilted potential. To see this from the model's spectrum, we first solve for the probability of being in odd sites and substitute the solution in the equation for the probability of being in even sites. The resulting coarse-grained equation [11], in the long-time limit, for the probability, $P(x, t)$, of being at site $x$ at time $t$ is given by

$$
\begin{aligned}
\left(w_{b} \vec{b}+w_{b}^{\leftarrow}\right) \partial_{t} P(x, t) & =w_{a}^{\rightarrow} w_{b} \vec{P} P(x-2, t)+w_{a}^{\leftarrow} w_{b}^{\leftarrow} P(x+2, t) \\
& -\left(w_{a}^{\overrightarrow{ }} w_{b} \vec{b}+w_{a}^{\leftarrow} w_{b}^{\leftarrow}\right) P(x, t)
\end{aligned}
$$

In the continuum limit, which describes the long time, large length-scale behavior of the model we have

$$
\partial_{t} P(x, t)=D \partial_{x}^{2} P(x, t)-v \partial_{x} P(x, t),
$$

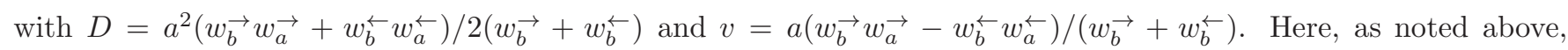
$a=2 a_{0}$ is a lattice constant analogous to a unit cell size in conventional solid state physics. We consider a system of size $L=N a$ and perform a Laplace transform in time and a Fourier transform in space so that

$$
P(x, t)=\sum_{k} g(k) e^{i k x+\lambda(k) t} .
$$


The coefficient $g(k)$ will depend on the initial conditions. If periodic boundary condition are imposed on a lattice of size $L$, the wavevectors $k$ specifying the (delocalized) eigenfunctions $\Psi_{k}(x) \sim e^{i k x}$ are quantized, $k_{n}=2 \pi n / L$, $n=0, \pm 1, \ldots$. In general, since the evolution operator is non-hermitian eigenvalues may be complex. The complex eigenvalues must come in complex conjugate pairs to ensure a real probability density $P(x, t)$ [19]. The eigenvalue spectrum is given by

$$
\lambda(k)=-D k^{2}-i v k
$$

corresponding to diffusion with drift. The dependence of the real part of the spectrum on $k$ describes diffusion, while the dependence of the imaginary part describes drift with a constant velocity. This identification is possible when periodic boundary conditions allow a current to flow in the system even in the limit of $t \rightarrow \infty$.

\section{B. Heterogeneous Model}

For the heterogeneous model the situation is more subtle, because the Fourier transform cannot be used to diagonalize the evolution operator. Nevertheless, we will show that a numerical analysis of the eigenvalue spectrum can be used to deduce the dynamical properties of the model. The role of the wave numbers in the homogeneous model is taken by winding numbers 28 .

The winding number, defined only for complex eigenfunctions, is given by the number of times the eigenfunction spirals around the origin in the complex plane as it traverses the whole lattice with periodic boundary conditions. For homogeneous systems and eigenfunctions which behave like $e^{i k_{n} x}$ we have $k_{n}=2 \pi n / N a$, implying a winding number $n$. For disordered systems, it can be shown [28] that the winding number increases linearly with the eigenvalue index $n$ which orders the eigenfunctions from the lowest value of $|\lambda(n)|$ to the highest. That is, the winding number associated with an eigenvalue of magnitude $|\lambda(n)|$ is $\pm n$. Moreover, if the eigenvalue $\lambda(n)$ is in the upper part of the complex $\lambda$ plane its winding number is $n$. Its complex conjugate pair $\lambda^{*}(n)$ will then have a winding number $-n$. For real eigenvalues the eigenfunctions can be chosen to be real and the winding number is not defined. In this case, the eigenfunctions can be classified by the number of zeros, as in one dimensional quantum mechanics [29].

The eigenvalue spectrum of the heterogeneous model with periodic boundary conditions can be used to encapsulate the long time dynamical properties of the model. Specifically, the dependence of the real and imaginary parts of the eigenvalues on the winding number signals the properties of the mean square displacement and drift of the motor molecule within our model.

To this end we consider the model of Sec. II with, as before, two types of monomers drawn at random. The set of parameters representing each type of monomer $\{p\}=\left\{\alpha, \alpha^{\prime}, \omega, \omega^{\prime}, \Delta \varepsilon\right\}$ is chosen with probability $1 / 2$ to be $\left\{p_{1}\right\}=\left\{\alpha_{1}, \alpha_{1}^{\prime}, \omega_{1}, \omega_{1}^{\prime}, \Delta \varepsilon_{1}\right\}$ and with probability $1 / 2$ to be $\left\{p_{2}\right\}=\left\{\alpha_{2}, \alpha_{2}^{\prime}, \omega_{2}, \omega_{2}^{\prime}, \Delta \varepsilon_{2}\right\}$. The chemical potential difference $\Delta \mu$ is assumed to be the same for both types of monomers. Allowing $\Delta \mu$ to depend on the type of monomer does not alter the qualitative long time behavior of the model.

We calculated the eigenvalue spectrum of the evolution operator numerically by diagonalizing a matrix with a specific realization of the disorder (see Appendix B for more details). From the exact solution of the model we expect three different regimes. Below a typical spectrum from each of the regimes is shown and examined in detail. Throughout we set $T=1$ and use the parameters $\left\{p_{1}\right\}=\left\{\alpha_{1}, \alpha_{1}^{\prime}, \omega_{1}, \omega_{1}^{\prime}, \Delta \varepsilon_{1}\right\}=\{6,1,1,6,0\}$ and $\left\{p_{2}\right\}=\left\{\alpha_{2}, \alpha_{2}^{\prime}, \omega_{2}, \omega_{2}^{\prime}, \Delta \varepsilon_{2}\right\}=\{1.2,1,1,1.2,0\}$ (again with equal probability) and $e^{\Delta \mu / T}=10$. The results are unchanged for similar sets of parameters. For these values of the parameters the exact locations of the transitions between the different regimes can be readily calculated using the results summarized in Sec. II.

Regime I: For $f<0.2256$ and $f>0.518$ the motion is biased diffusion. A typical spectrum of eigenvalues in the complex plane (for $f=0$ ) is shown in Fig. 17. We show only the eigenvalues with the smallest $|\lambda|$, because those with larger values are non-universal and are highly dependent on the details of the model used to describe the motion of the motor. The spectrum has two branches in the complex $\lambda$ plane. Also, it has no purely real parts (with the exception of $\lambda=0)$.

For usual diffusion with uniform drift in a homogeneous system, as discussed above, $\lambda(k)=-D k^{2}-i v k$. The imaginary part of the spectrum corresponds to drift, the real part to diffusion. A similar behavior might be expected in our heterogeneous model for $\lambda(k)$ where $k$ is related to the winding number as $k=k_{n}=2 \pi n / N a$. This can be seen in a plot of the real and imaginary parts of the spectrum as a function of the eigenvalue index as defined above. Since in this regime the dynamics is biased diffusion we expect $\operatorname{Im}(\lambda(n)) \propto n$, while $\operatorname{Re}(\lambda(n)) \propto n^{2}$.

To test this hypothesis, we order the eigenvalues according to their magnitude and $\operatorname{plot} \operatorname{Im}(\lambda(n))$ and $\operatorname{Re}(\lambda(n))$. Fig. 8 shows $\operatorname{Im}(\lambda(n))$ for small $n$. One can see that indeed the slope in linear. Fig. 9] shows $-\operatorname{Re}(\lambda(n))$ for $\operatorname{small} n$ on a $\log -\log$ scale along with a line $-\operatorname{Re}(\lambda(n))=A n^{2}$ with $A$ some constant. At small $n$ indeed $\operatorname{Re}(\lambda(n)) \propto n^{2}$ for more than a decade. 


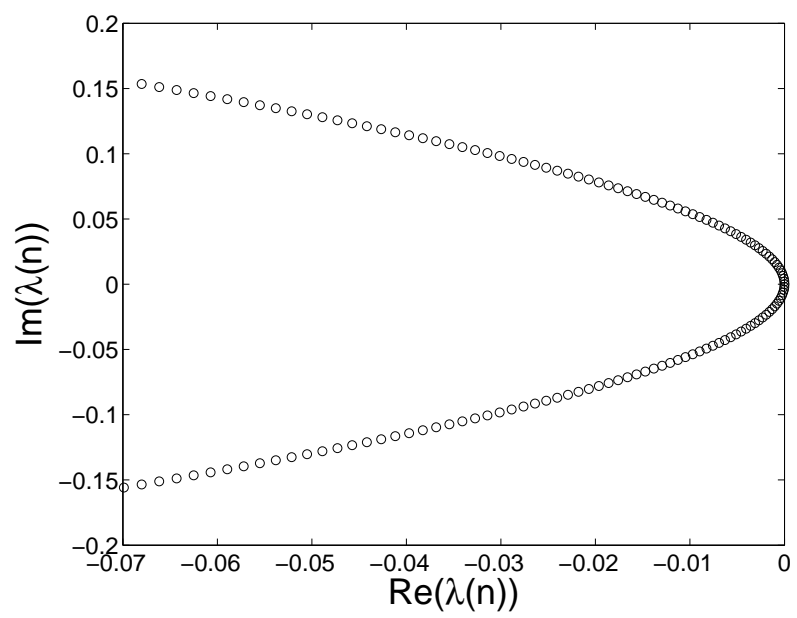

FIG. 7: The eigenvalues obtained for $f=0$. Here the system size is $N=4500$. Shown are the 140 eigenvalues with the lowest value of $|\lambda|$. In the region near the origin we expect that $\operatorname{Im}(\lambda) \propto \pm \sqrt{|\operatorname{Re}(\lambda)|}$.

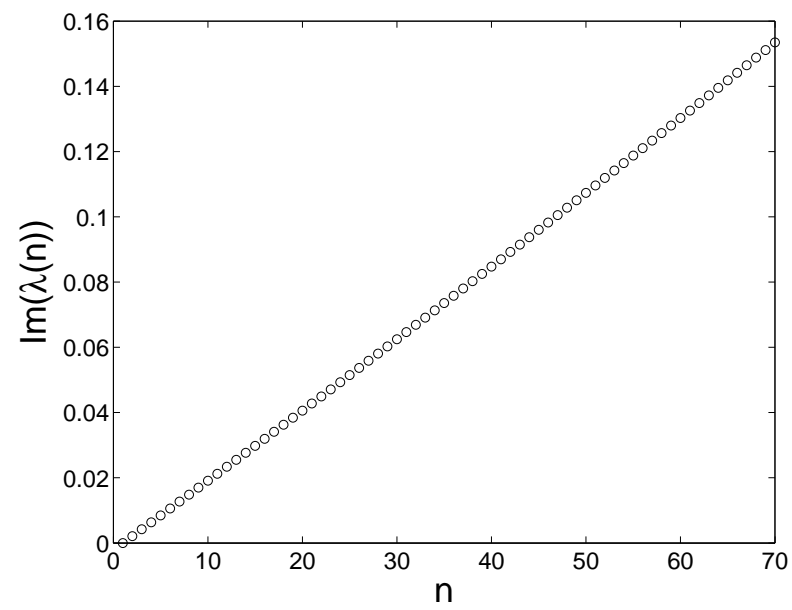

FIG. 8: The imaginary part of the eigenvalue, $\operatorname{Im}(\lambda(n))$ for $f=0$ as a function of $n$. Here the system size is $N=4500$. Shown are the 140 eigenvalues with the lowest value of $|\lambda|$.

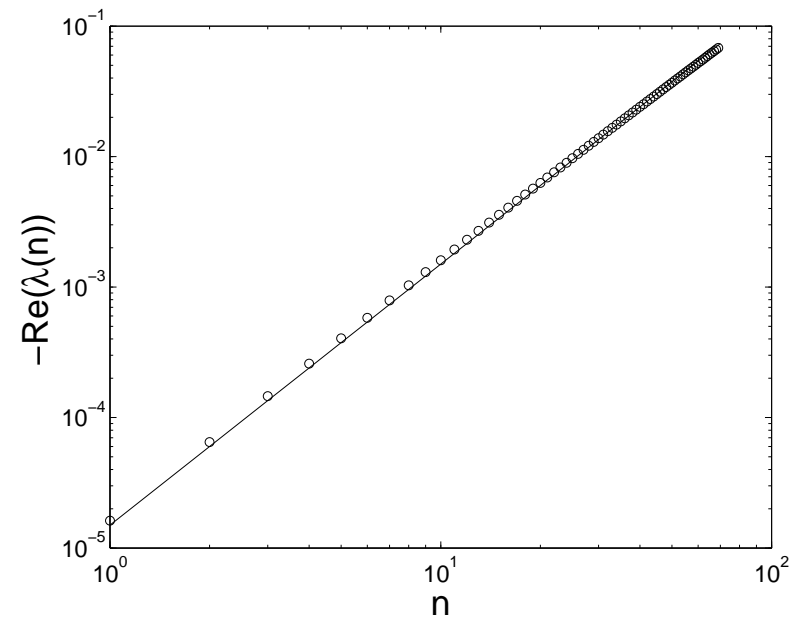

FIG. 9: The real part of the eigenvalue, $-\operatorname{Re}(\lambda)$ for $f=0$ as a function of the winding number $n$. Here the system size is $N=4500$. Shown are the 140 eigenvalues with the lowest value of $|\lambda|$. The solid line is the function $A n^{2}$ with $A$ a constant. 


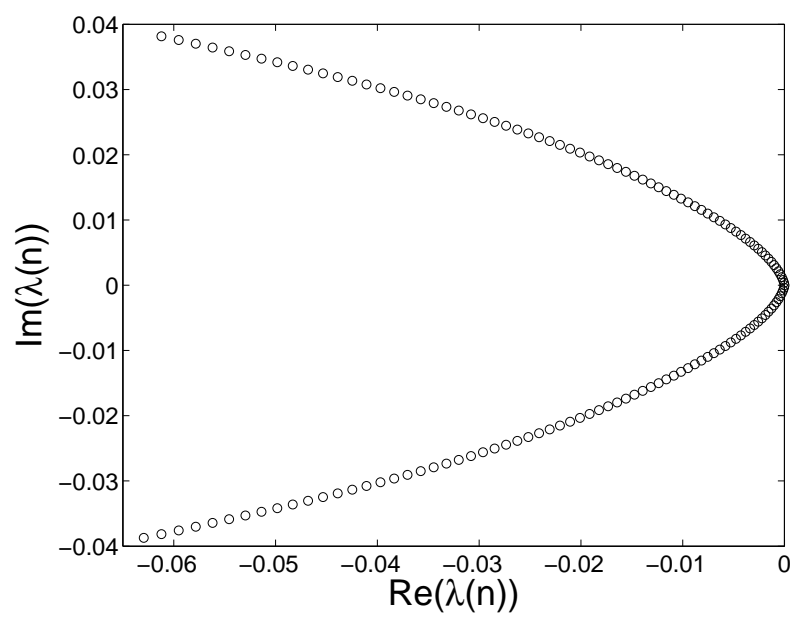

FIG. 10: The eigenvalues obtained for $f=0.25$. Here the system size is $N=4500$. Shown are the 140 eigenvalues with the lowest value of $|\lambda|$. Near the origin we expect that $\operatorname{Im}(\lambda) \propto \pm|\operatorname{Re}(\lambda)|^{1 / \mu}$, with $1<\mu<2$.

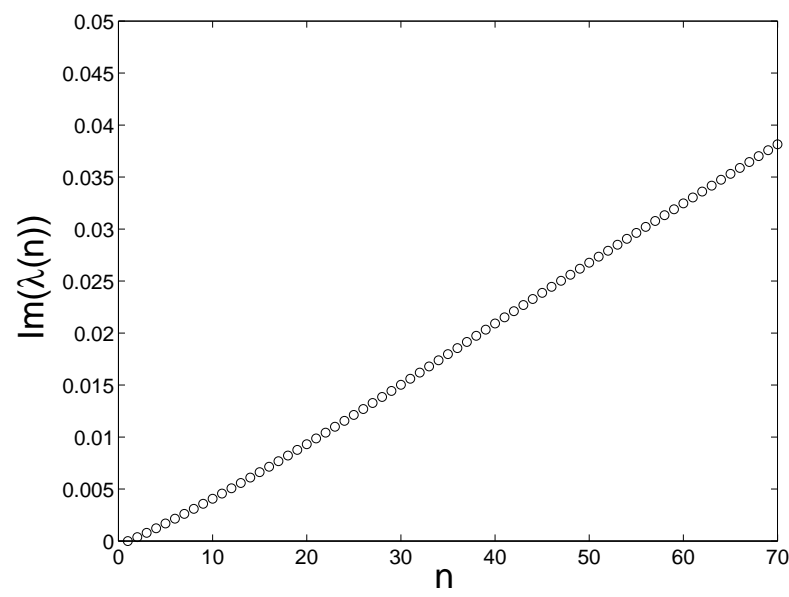

FIG. 11: The imaginary part of the eigenvalue for $f=0.25$ as a function of the winding number $n$. Here the system size is $N=4500$. Shown are the 140 eigenvalues with the lowest value of $|\lambda|$.

Regime II: Next we turn to look at the region where the diffusion constant depends on the size of the time averaging window used to evaluate it, while the velocity does not. For the parameters used in the numerics this occurs for force windows given by $0.2256<f<0.2882$ and $0.4555<f<0.518$. A typical spectrum of eigenvalues in the complex plane is shown in Fig. 10 Again we concentrate on the small $|\lambda|$ part of the spectrum and examine $\operatorname{Im}(\lambda(n))$ and $\operatorname{Re}(\lambda(n))$. Fig. 11]presents a plot of $\operatorname{Im}(\lambda(n))$ for small $n$. The expected dependence on $n$ is linear. A careful analysis of the curve shows that $\operatorname{Im}(\lambda(n))$ can be fit well to $A n+B n^{3}$ for small $n$ (here we have used that fact that $\operatorname{Im}(\lambda(n)$ ) is expected to be an odd function of $n)$. The coefficient $B$ decreases as the size of the system studied is increased. Such a correction is expected if the time to reach the asymptotic behavior $\langle x\rangle \sim v t$ is so large that it is comparable to the relaxation time of the system. For larger systems the correction will become less important and the asymptotic behavior will be observed more easily. Fig. 12] shows $-\operatorname{Re}(\lambda(n))$ for small $n$ on a $\log$-log scale along with the lines $A n$ and $B n^{2}$ with $A$ and $B$ some constants. At small $n, \operatorname{Re}(\lambda(n))$ indeed does not behave as $n^{2}$. It shows a smaller slope in accordance with a behavior consistent $n^{\mu}$ with $1<\mu<2$. In fact, from naive dimensional considerations $(\lambda$ behaves as $1 / t$ while $x \sim n$ ) this is the behavior expected when $\left\langle x^{2}\right\rangle-\langle x\rangle^{2} \sim t^{2 / \mu}$.

Regime III: Finally, we turn to look at the region where the velocity is effectively zero as $t_{W} \rightarrow \infty$ and the diffusion is again anomalous. For the parameters used in our numerical analysis this regime occurs for $0.2882<f<0.518$. A spectrum of eigenvalues in the complex plane is shown in Fig. [13] Again we concentrate on the small $|\lambda|$ part of the spectrum. To get a clear picture one needs to plot both $\operatorname{Im}(\lambda(n))$ and $\operatorname{Re}(\lambda(n))$. The positive part $\operatorname{Im}(\lambda(n))$ is plotted on a log-log plot in Fig. 14 Here the displacement of the particle is expected to behave as $t^{\mu}$ with $\mu<1$. This leads to an expected dependence $\operatorname{Im}(\lambda(n)) \propto n^{1 / \mu}$. A reference line $A n$ with $A$ a constant is plotted for comparison. 


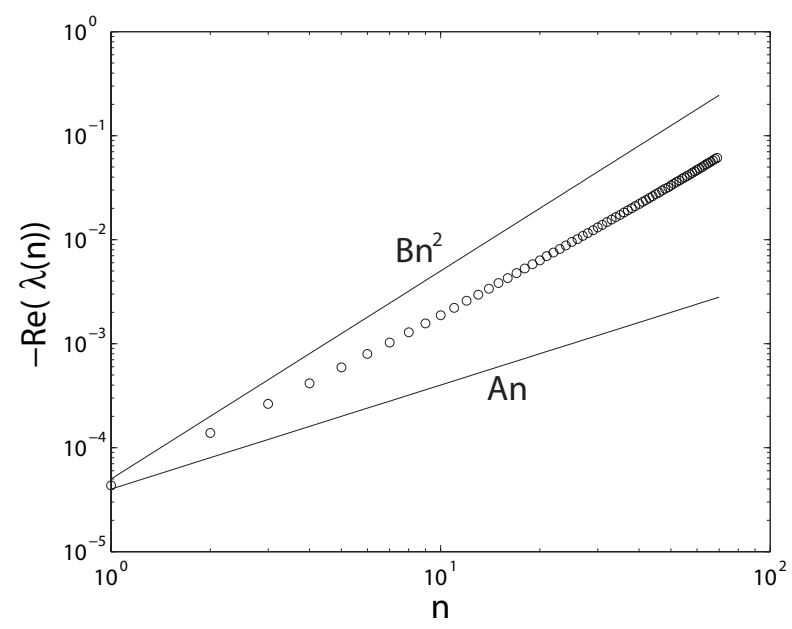

FIG. 12: The real part of the eigenvalue, $-\operatorname{Re}(\lambda)$ for $f=0.25$ as a function of $n$. Here the system size is $N=4500$. Shown are the 140 eigenvalues with the lowest value of $|\lambda|$. The function $A n$ and $B n^{2}$ with $A$ and $B$ constants are plotted for reference. From a fit to the slope of our numerical data we find $\mu=1.915 \pm 0.004$.

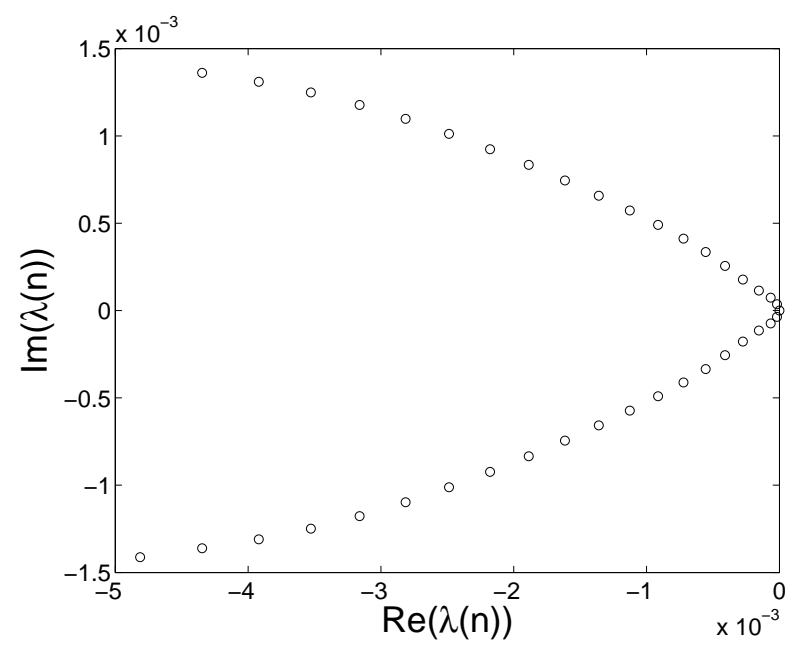

FIG. 13: The eigenvalue spectrum obtained for $f=0.31$, where both diffusion and drift are anomalous. Here the system size is $N=4500$. Shown are the 40 eigenvalues with the lowest value of $|\lambda|$. Near the origin, we $\operatorname{expect} \operatorname{Im}(\lambda) \propto \pm|\operatorname{Re}(\lambda)|$.

Clearly, $\operatorname{Im}(\lambda(n))$ behaves as expected.

Fig. 115 shows $-\operatorname{Re}(\lambda(n))$ for small $n$ on a $\log$-log scale along with a line $A n^{2}$ with $A$ some constant. As can be seen at small $n$ indeed $\operatorname{Re}(\lambda(n))$ does not behave as $n^{2}$. Here a similar argument as before leads one to expect $-\operatorname{Re}(\lambda(n)) \propto n^{1 / \mu}$ for small $n$, consistent with the numerics.

We comment that the analysis is only possible not too far from the transition point into regime III. Deep inside the region with anomalous velocity, the small $|\lambda|$ spectrum becomes very noisy due to finite size effects. Analysis of the spectrum then becomes difficult due to these strong sample-to-sample fluctuations. Hence we have not attempted an analysis in the Sinai diffusion regime, where the energy landscape is nearly horizontal.

\section{FINITE PROCESSIVITY}

\section{A. Homogeneous Model}

As discussed above, the homogeneous model is well described on long time scales and large length scales by a random walker moving along a tilted potential. The unbinding of motors from the track adds a non-conserving term 


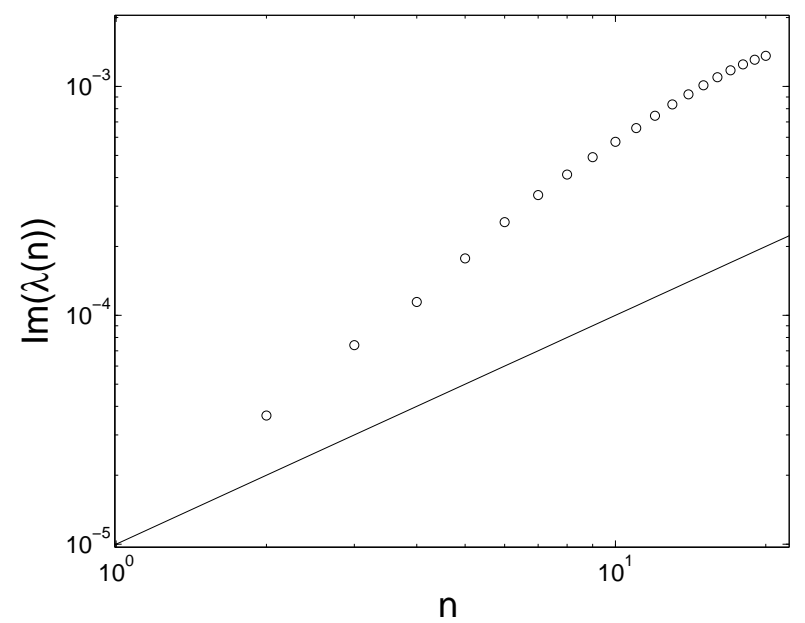

FIG. 14: The imaginary part of the eigenvalue for $f=0.31$ as a function of the winding number $n$. Here the system size is $N=4500$. Shown are the 40 eigenvalues with the lowest value of $|\lambda|$. A fit to $\operatorname{Im}(\lambda) \propto-|n|^{1 / \mu}$, over only one decade, gives $\mu=0.6 \pm 0.1$. The function $A n$ with $A$ a constant is plotted for reference.

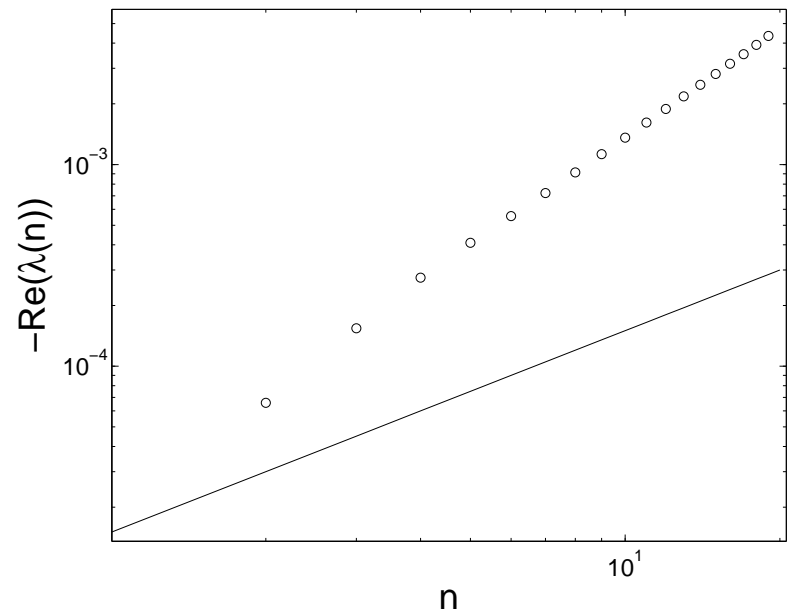

FIG. 15: The real part of the eigenvalue, $-\operatorname{Re}(\lambda)$ for $f=0.31$ as a function of $n$. Here the system size is $N=4500$. Shown are the 40 eigenvalues with the lowest value of $|\lambda|$. A fit to $\operatorname{Re}(\lambda) \propto n^{1 / \mu}$, over only one decade, gives $\mu=0.6 \pm 0.1$. The function An with $A$ a constant is plotted for reference.

to the equation for the probability density. In a continuum description, one now has

$$
\partial_{t} P(x, t)=D \partial_{x}^{2} P(x, t)-v \partial_{x} P(x, t)-w_{\text {off }} P(x, t) .
$$

In terms of the microscopic model as before one has $D=a^{2}\left(w_{b} \vec{b} w_{a}+w_{b}^{\leftarrow} w_{a}^{\leftarrow}\right) / 2\left(w_{b} \vec{t}+w_{b}^{\leftarrow}\right)$ and $v=a\left(w_{b} \vec{b} w_{a}^{\rightarrow}-\right.$ $\left.w_{b}^{\leftarrow} w_{a}^{\leftarrow}\right) /\left(w_{b} \vec{t}+w_{b}^{\leftarrow}\right)$. In addition, here $w_{\text {off }}=\left(w_{\text {off }}^{b}\left(w_{a}^{\rightarrow}+w_{a}^{\leftarrow}\right)+w_{\text {off }}^{a}\left(w_{b} \vec{b}+w_{b}^{\leftarrow}\right)\right) /\left(w_{b}+w_{b}^{\leftarrow}\right)$

Again, we consider the model with periodic boundary conditions and perform a Laplace transform in time and a Fourier transform in space. The eigenvalue spectrum is given by $\lambda(k)=-D k^{2}-i v k-w_{\text {off }}$. When $w_{\text {off }}=0$ the motion is diffusion with drift. However, when $w_{\text {off }}>0$ the probability density decays exponentially to the empty track state $P(x, t)=0$ with a typical time scale $1 / w_{\text {off }}$. Note, however, that the eigenfunctions are identical to the infinite processivity case. In fact, the probability density of the motor can be written as

$$
P(x, t)=e^{-w_{\text {off }} t} P_{w_{\text {off }}=0}(x, t) .
$$

Up to a rescaling of the probability density the time evolution is unaltered. That is, the motors which remain on the track are unaffected by a constant hopping off rate. 


\section{B. Heterogeneous Model}

If the hopping off rates are uniform along the track, but the remaining parameters are chosen randomly, $w_{\text {off }}^{a}$ and

$w_{\text {off }}^{b}$ are the same for all types of monomers, and it is straightforward to see that the effect is the same as for a homogeneous system: the dynamics of the motors which remain on the track are unaffected by the non-conservation.

We now show that, when randomness in the rates for hopping off the track is introduced into the system, the dynamics of the motors which remain on the track can be altered in a dramatic way. The eigenfunctions of the evolution operator are affected by the heterogeneous hopping off rates as well as by the sequence heterogeneity as it affects the local diffusion constant and drift velocity. As we shall see, disorder in the hopping off rates has a profound effect on the behavior of motors which remain on the track. We stress that, with applications to molecular motors in mind, the disorder in the detachment rates we consider is correlated with the local hopping rates.

As for the conserving heterogeneous model with its random force landscape, we study the dynamics by considering the spectral properties of the evolution operator of the model. The effect of detachment is included by setting the rates $w_{\text {off }}^{a}$ and $w_{\text {off }}^{b}$, corresponding to leaving the track at even and odd sites respectively, to be non-zero and site dependent. We find two types of behavior which depend on the drift properties of the motor for the corresponding conserving model. When the drift is linear in time the last motors to be left on the track localize only beyond a critical disorder strength in the hopping off rates. Similar behavior arises in the physics of vortices [18, 19] and in population dynamics 27]. The behavior when the displacement of the motor for the conserving model is sublinear (i.e. when it is not possible to define a drift velocity) is different. Now, the last motors to remain on the track are always localized for any strength of the disorder.

\section{Ballistic displacement}

First, we consider the regime where the displacement of the motor in the conserving model is linear as a function of time $(\mu>1)$. We will show that when the disorder in the rates of leaving the track is strong enough the eigenvalues with the smallest $|\lambda|$ become purely real. This is a regime of localized eigenfunctions and corresponds to motors with zero drift velocity. This feature is present over the whole region where $\mu>1$.

To explore this behavior, we have studied the eigenvalue spectrum of the model numerically for systems of size 4500. In Fig. [16] results are shown for the case $f=0(\mu>2)$. However, the localization effects which are of interest to us here remain unchanged for all values of $f$ such that $\mu>1$. The parameters used are the same as used previously in the paper augmented by $w_{\mathrm{off}}^{a}=0.01$ and $w_{\mathrm{off}}^{b}=0.01\left(w_{\mathrm{off}}^{a}=0.02\right.$ and $\left.w_{\mathrm{off}}^{b}=0.02\right)$ for the parameter set $\left\{p_{1}\right\}$ $\left(\left\{p_{2}\right\}\right)$. As can be seen, except for the lowest $|\lambda|$ eigenvalue, all eigenvalues have an imaginary part. Thus, the motors are biased to move across the lattice in a positive direction. In Fig. 17 we show the spectrum with $w_{\text {off }}^{a}=0.01$ and $w_{\text {off }}^{b}=0.01\left(w_{\text {off }}^{a}=0.08\right.$ and $\left.w_{\text {off }}^{b}=0.08\right)$ for the set $\left\{p_{1}\right\}\left(\left\{p_{2}\right\}\right)$, which corresponds to a larger disorder strength. Here eigenvalues beyond a certain mobility edge become real, implying that the long lasting motors are described by localized states 19, 26. Note, that the disorder in the hopping off rates is correlated with the disorder in the hopping rates themselves, as required if both are due to the same underlying heterogeneous polynucleotide sequence.

The transition between moving and localized motors can be understood, in the case of ballistic displacement, by adapting the ideas of Ref. [30]. We consider a simplified model for the motors. The energy landscape consists of two slopes $s_{1}$ and $s_{2}$ (corresponding to two types of monomers). The velocity on a track with a slope $s_{1}\left(s_{2}\right)$ is denoted be $v_{1}\left(v_{2}\right)$. The rate for leaving the track on a region with a slope $s_{1}$ is assumed to be $w$, while the rate for leaving the track on a region with slope $s_{2}$ is 0 . Note that as before the local detachment rates are correlated with the local biases.

Next, compare two "survival" strategies for the motor in the long time limit. In the first it spreads across the lattice probing regions with slope $s_{1}$ and $s_{2}$. In the other it remains in regions with slope $s_{2}$ where detachment rates are zero. The probability of staying in such regions can be estimated by solving for a random walker moving on a tilted potential of some size with absorbing boundary conditions at both ends. One finds [30] that this probability behaves as

$$
P_{\text {stay }}(t) \sim e^{-v_{2}^{2} t / 4 D},
$$

where $D$ is the diffusion coefficient on a track with slope $s_{2}$. The probability of survival of a particle moving across the lattice can also be evaluated. One expects

$$
P_{\text {move }}(t) \sim e^{-c w t},
$$

where $c$ is a constant which will depend on the disorder averaged velocity. For large enough $w$, the particles that choose the localized strategy clearly will have a better chance of survival. One therefore expects that as the disorder 


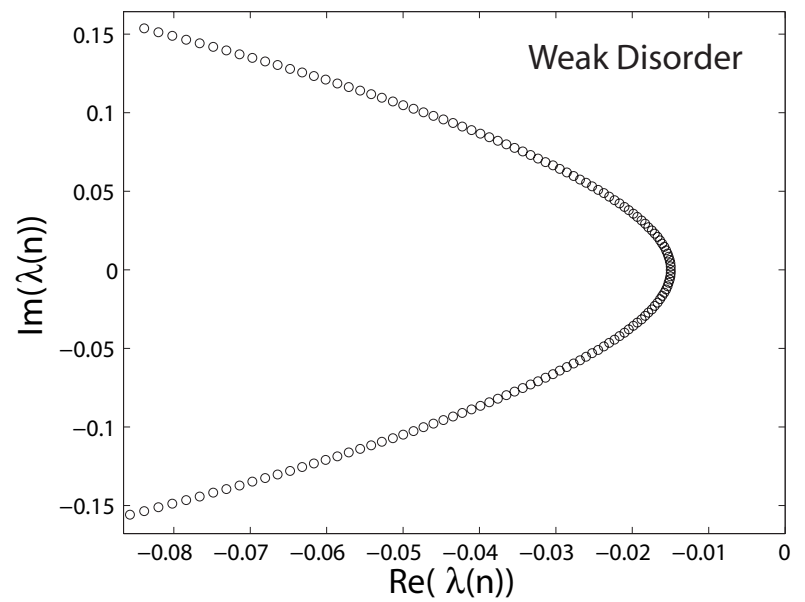

FIG. 16: The eigenvalue spectrum for $f=0$ and $\mu>2$ with rates for hopping off the track as specified in the text. Except for the largest eigenvalue, all eigenvalues have an imaginary part. All eigenfunctions, moreover describe extended states with a well-defined winding number. Shown are the 140 eigenvalues with the lowest value of $|\lambda|$ and the system size is $N=4500$.

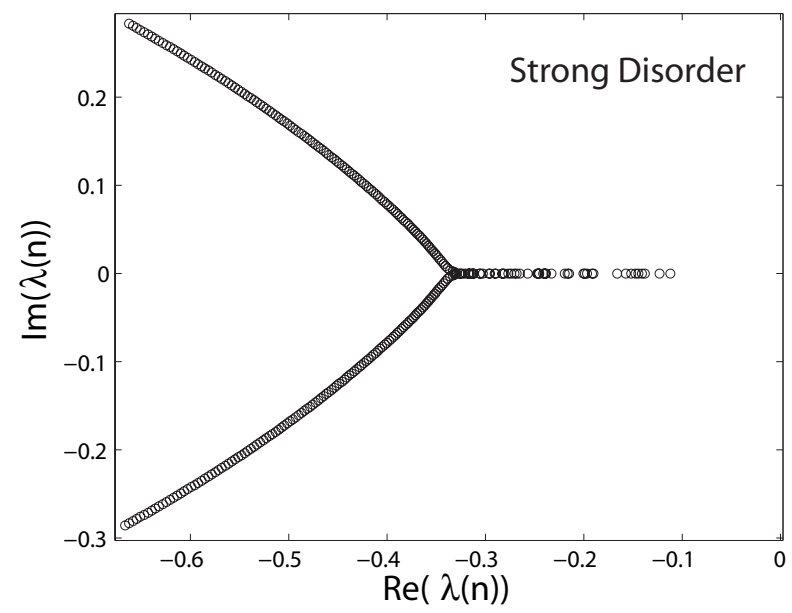

FIG. 17: The eigenvalue spectrum for $f=0$ with rates for hopping off the track as specified in the text. As can be seen the eigenvalues with the lowest value of $|\lambda|$ become localized. Shown are the 300 eigenvalues with the lowest value of $|\lambda|$ and the system size is $N=4500$.

in the hopping off rates is increased a transition from localized states to delocalized states will occur at the high end of the spectrum (corresponding to small $k$ and small winding numbers). Note that the argument would not be altered if the rate for hopping off from negative slope regions were non-zero, although, the transition point between the two regimes would be shifted.

Finally we comment that this argument could also be used for a model with a random energy landscape (see Eq. 44. This case is very similar to problems which have been studied in the context of the vortex physics [18, 19] and population dynamics [26]. For completeness, Appendix C presents eigenvalue spectra, for weak and strong disorder, in that case.

\section{Sublinear displacement}

A typical spectrum in the regime of sublinear displacement, $\mu<1$, is shown in Fig. 18 Note the small band of localized states with real eigenvalues near the top of the spectrum (small $|\lambda|$ ). Again the parameters used are the same as used previously with the falling from the track rates $w_{\mathrm{off}}^{a}=0.01$ and $w_{\mathrm{off}}^{b}=0.01\left(w_{\mathrm{off}}^{a}=0.02\right.$ and $\left.w_{\mathrm{off}}^{b}=0.02\right)$ for the set $\left\{p_{1}\right\}\left(\left\{p_{2}\right\}\right)$. Note, that even for these small values of the falling off rates localized states appear. This effect persists even for falling off rates smaller by more than an order of magnitude and for various values of $f$ in 


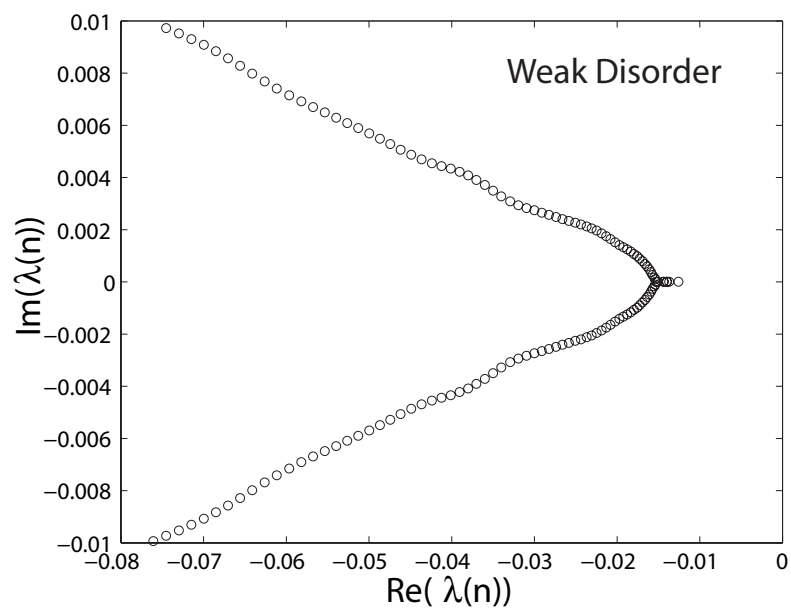

FIG. 18: The eigenvalue spectrum for $f=0.31$ with rates for hopping off the track as specified in the text. The small band of real eigenvalues at the top of the spectrum characterizes localized states. Shown are the 140 eigenvalues with the lowest value of $|\lambda|$, and the system size is again $N=4500$.

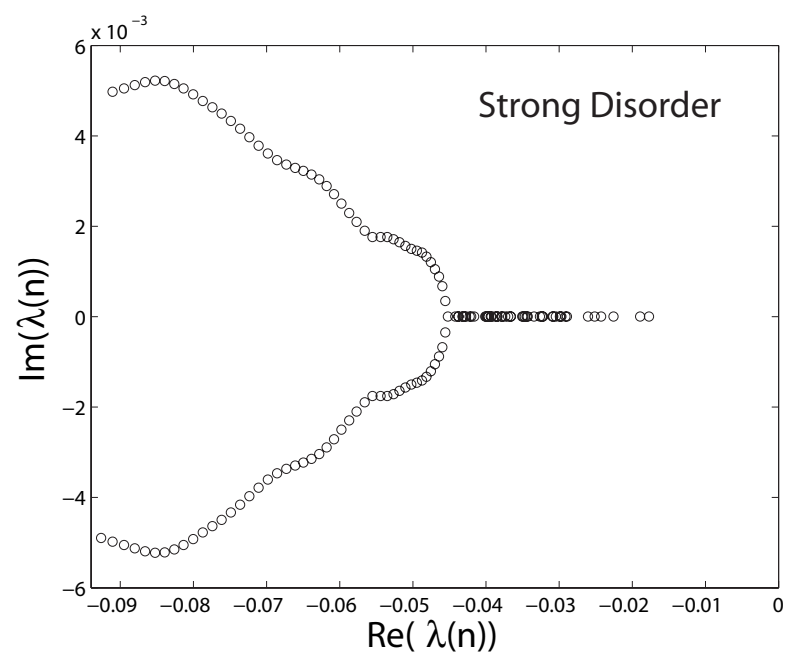

FIG. 19: The eigenvalue spectrum for $f=0.31$ with rates for hopping off the track as specified in the text. The spectrum is qualitatively similar to that of Fig. [18 with weak disorder. The band of real eigenvalues at the top of the spectrum characterizes localized states and now contains, as expected, more states than Fig. 18 Shown are the 140 eigenvalues with the lowest value of $|\lambda|$, and the system size is again $N=4500$.

this regime as long as large enough systems were studied. For comparison Fig. 19 shows the spectrum in the case of strong disorder. Here $w_{\text {off }}^{a}=0.01$ and $w_{\text {off }}^{b}=0.01\left(w_{\text {off }}^{a}=0.06\right.$ and $\left.w_{\text {off }}^{b}=0.06\right)$ for the parameter set $\left\{p_{1}\right\}\left(\left\{p_{2}\right\}\right)$. As always in this paper, the two parameter sets are assumed t ooccur with equal probability. As can be seen from the figure, while details of the spectrum are different from the weak disorder case shown in Fig. 18 they are qualitatively the same.

\section{Implications for the probability of finding a motor on the track as a function of time}

Next, we discuss the effect of the localized states occurring near the top (low $|\lambda|$ ) of the spectrum on the probability of finding a motor on the track as a function of time. It may be possible to reveal these localized states by looking at the density decay as a function of time of a dilute concentration of fluorescently labeled motors placed on a track. The probability of finding a motor on the track as a function of time, $P_{s}(t)$, could then be monitored by looking at the decay of the fluorescent signal as a function of time (possibly averaging over several experiments). 
The implication of localized states for the probability $P_{s}(t)$ has already been considered in the context of random walkers subject to the influence of random traps [30]. The arguments are unaltered for the case of a random forcing energy landscape, and here we consider a simple version. A more detailed proof can be carried out along the lines of Ref. [26]. We consider a system where the hopping and detachment rates can only assume two values. We are interested in the behavior in the long-time limit. When localized states exist the eigenfunctions are strongly peaked in regions where the probability of detachment from the track is small. The probability for such a region of length $l$ to occur behaves as $e^{-\gamma l}$ where $\gamma$ is a constant. On the other hand the time to leave the region behaves as $e^{-v_{2}^{2} t / 4 D+\chi D t / l^{2}}$, where $\chi$ is a constant of order unity and we have included higher order corrections to Eq. 17 associated with the finite size $l$ of the region. Note that we have used the fact that the detachment rate is correlated with the local hopping rate so that the behavior inside the region is diffusive irrespective of the value of $\mu$. Summing over the contribution from regions of different lengths one obtains

$$
P_{s}(t) \sim e^{-v_{2}^{2} t / 4 D-\left(t / t_{0}\right)^{1 / 3}} .
$$

Here $t_{0}$ depends on the constants $\gamma$ and $\chi$ and $D$ the diffusion coefficient. As expected the leading behavior is still exponential. However, a signature of the localized states appears in the correction which has a stretched exponential form.

In contrast, when no localized states exist the behavior is dominated by the detachment rates. In that case no diffusive corrections to the detachment rate from a region of size $l$ are present. The stretched exponential correction to the decay of $P_{s}(t)$ displayed in Eq. (19) will be absent.

In summary, in the region of sublinear displacement one expects a stretched exponential behavior to always be present at long times when the probability of finding a motor on the track is monitored. In the ballistic displacement regime the correction will be present only for strong enough disorder in the detachment rates.

\section{TOY MODEL}

The understanding of the dynamics of random walkers (with infinite processivity) on random forcing energy landscapes has been enhanced by a simple toy model introduced by Bouchaud et. al. 12. The model builds on the fact that at finite tilt the sojourn time $\tau$ at any site is found to have a broad distribution $\Psi(\tau) \sim \tau^{-(1+\mu)}$ for large $\tau$. This behavior suggests that the dynamics could be mimicked by a directed walk between traps with a broad release time distribution. Specifically, the model consists of a particle moving unidirectionally on a lattice with hopping rates $W_{k}$ between site $k-1$ and $k$ which are drawn from a probability distribution which satisfies $\Psi(W) \approx \bar{A} W^{\mu-1}$ as $W \rightarrow 0$. Although the equivalence to the original model cannot be justified rigorously (since back stepping is ignored), the two models are known to exhibit the same long time behavior.

Here we analyze the model from a somewhat different perspective and show that indeed it yields for the infinite processivity limit the types of spectra described in Section III. Moreover, we study the finite processivity model in the limit of weak disorder in the rates for leaving the track. Here the rate for leaving the track at site $k, w_{k}$, is assumed to be uncorrelated with the hopping rate $W_{k}$. If one views the directed model as a coarse-grained version of the original undirected model, then one expects that the hopping rates $W_{k}$ are determined by the depth of the traps in the energy landscape. In contrast, since the particles spend most of their time near the bottom of the traps, the coarse-grained off rates in the directed model will depend primarily on the local off rates near the trap bottoms in the original model, suggesting that the $w_{k}$ and $W_{k}$ should indeed be uncorrelated. With this simplification, we show that similar to the more complex models discussed earlier in this paper, any strength of disorder in the rates for leaving the lattice causes the eigenfunctions describing the long-time behavior of the model to be localized for $\mu<1$. In contrast for $\mu>1$, weak disorder leaves the eigenfunctions delocalized.

It is convenient to first consider a more general model with non-zero rates for leaving the track. We derive an equation for the eigenvalue spectrum of the model and then analyze the spectra separately in the infinite and finite processivity limits.

The time evolution of the model is described by the set of master equations

$$
\frac{d P_{k}(t)}{d t}=W_{k} P_{k-1}-W_{k+1} P_{k}-w_{k+1} P_{k} .
$$

After a Laplace transform, the corresponding equations for the eigenfunctions is then

$$
\left(\lambda+W_{k+1}+w_{k+1}\right) P_{k}(\lambda)=W_{k} P_{k-1}(\lambda)
$$


which yields a recursion relation for the $P_{k}(\lambda)$ which can be readily solved. The possible eigenvalues $\lambda$ are then determined by imposing periodic boundary conditions, yielding

$$
\begin{aligned}
1 & =\prod_{i=1}^{N} \frac{W_{i}}{\left(\lambda+W_{i}+w_{i}\right)} \\
& \sim \exp \left(N \overline{\left(\ln \frac{W}{\lambda+W+w}\right)}\right)
\end{aligned}
$$

where the last line holds in the limit when the system size $N$ is taken to infinity and the overline denotes as before average over disorder. Care must be taken when $\lambda$ is real. In this case the recursion relation might allow for some $\lambda+W_{k+1}+w_{k}$ to be zero. In that case $P_{k}(\lambda)$ can assume any value (to be set by normalization), while $P_{k-1}(\lambda)=0$. Such eigenfunction will be referred to as "localized", with a detailed justification given below. When $\lambda$ is complex, we refer to the eigenfunctions as delocalized.

According to Eq. [22], we need to calculate

$$
R(\lambda)=\overline{\left(\ln \frac{W}{\lambda+W+w}\right)} .
$$

Since we are considering the long time behavior and small rates for leaving the track we consider the limit $|\lambda+w| \ll 1$. It will be useful to hold $w$ fixed for now and average over it later. We denote the function obtained by averaging only over $W$ in Eq. (23) by $Q(\lambda, w)$. We find that it depends on the value of the exponent $\mu$ as follows:

Case I: $\mu>2$. Here one finds to leading order in $(\lambda+w)$ that

$$
Q(\lambda, w)=-\overline{\left(\frac{1}{W}\right)}(\lambda+w)+\overline{\left(\frac{1}{W^{2}}\right)} \frac{(\lambda+w)^{2}}{2} .
$$

Case II: $1<\mu<2$. Here $\overline{W^{-2}}$ diverges and one has

$$
Q(\lambda, w)=-\overline{\left(\frac{1}{W}\right)}(\lambda+w)+B(\lambda+w)^{\mu},
$$

where $B=\bar{A} \pi / \mu \sin (\pi \mu)$. Here, $\bar{A}$ is the amplitude of the tail of the probability distribution, $\Psi(W) \approx \bar{A} W^{\mu-1}$.

Case III: $\mu<1$. Here both $\overline{W^{-1}}$ and $\overline{W^{-2}}$ diverge and one has

$$
Q(\lambda, w)=B(\lambda+w)^{\mu} .
$$

With these results at hand we now turn to analyze the infinite processivity limit.

\section{A. Infinite Processivity limit}

In this case we set $w=0$ for all sites. To analyze the eigenvalue spectrum we rewrite Eq. (22) in the form

$$
R(\lambda)=2 \pi i n / N \text {. }
$$

Consider the solution for the different cases

Case I: $\mu>2$. Here one has

$$
2 \pi i n / N=-\overline{\left(\frac{1}{W}\right)} \lambda+\overline{\left(\frac{1}{W^{2}}\right)} \frac{\lambda^{2}}{2} .
$$

It is straight forward to solve the quadratic equation for $\lambda$ and realize that $\operatorname{Im}(\lambda) \propto n$ while $\operatorname{Re}(\lambda) \propto n^{2}$ in agreement with the numerics of section

Case II: $1<\mu<2$. In this regime one has to solve

$$
2 \pi i n / N=-\overline{\left(\frac{1}{W}\right)}(\lambda)+B(\lambda)^{\mu}
$$

where $B=\bar{A} \pi / \mu \sin (\pi \mu)$. Again, a straightforward analysis shows that $\operatorname{Im}(\lambda) \propto n$ while $\operatorname{Re}(\lambda) \propto n^{\mu}$ in agreement with the numerics.

Case III: $\mu<1$. Finally, in this case one has

$$
2 \pi i n / N=B(\lambda)^{\mu}
$$

which evidently gives $\operatorname{Im}(\lambda) \propto n^{1 / \mu}$ and $\operatorname{Re}(\lambda) \propto n^{1 / \mu}$. 


\section{B. Finite Processivity}

Next, we turn to the finite processivity case. Here it will be sufficient to consider only the leading order contribution to $R(\lambda)$. To simplify, we study the case where $w$ can take only two values $w_{1}=w$ and $w_{2}=0$, which occur with probability $p_{1}$ and $p_{2}$. The general case can be analyzed very similarly. This assumption gives, after performing the average over $w$,

$$
R(E)=p_{1} Q(E, w)+p_{2} Q(E, 0) .
$$

As in the previous subsection we analyze behavior of the solutions of the equations for $\lambda$ and $w$ small. Here it will only be necessary to consider the cases $\mu>1$ and $\mu<1$.

Case I: $\mu>1$. Here we have

$$
-\left\langle\frac{1}{W}\right\rangle\left(p_{1}(\lambda+w)+p_{2} \lambda\right)=2 \pi n i / N
$$

with $n$ an integer. Clearly, to solve the equation $\lambda$ must have an imaginary part. (Note that we are missing the dependence of the real part of the solution on $n$ since we neglected higher order terms in $\lambda$ ). An imaginary part of the eigenvalue implies that delocalized states exist for small disorder in $w[19]$.

Case II: $\mu<1$. Here one has

$$
B\left(p_{1}(\lambda+w)^{\mu}+p_{2} \lambda^{\mu}\right)=\frac{2 \pi n i}{N}
$$

Consider first the case $n=0$. In this case the eigenvalue must be real [31] and since the decay of particles from the system can not be faster than $e^{-w t}$ one must have $-w \leq \lambda<0$. Clearly, such a solution can not exist. Thus, the assumption leading to Eq. (33), that none of the $P_{k}(\lambda)$ is zero, fails. As we argued early, $P_{k}(\lambda)=0$ away from a particular site, can only occur when $\lambda$ is real. It is possible to show that in this case the density is peaked near the site where $P_{k}(\lambda)=0$ decaying exponentially fast as $k$ increases. We conclude that for any strength of disorder in the hopping off rates the eigenfunctions become localized. The lack of solution for cases with $n>0$ can be proved by expanding around the lowest $|\lambda|$ (real) eigenvalue and discovering that a solution is impossible.

Acknowledgments: DKL and DRN thank Thomas Franosch for helpful discussions during the early stages of this investigation. DKL also benefitted from discussion with Piet Brouwer and Christopher Mudry. Work by YK and DRN was supported by the National Science Foundation through Grant DMR-0231631 and the Harvard Materials Research Laboratory via Grant DMR-0213805. YK was also supported through Grant DMR-0229243.

\section{APPENDIX A: SIMULATIONS}

In the following we describe briefly the procedure we used to simulate the model Eq. (10. To make the simulation efficient we first normalize the rates so that the largest one is equal one. Then, at each step we choose with equal probability between moving the motor to the right or left on the lattice. Following this choice a random number is drawn from a uniform distribution. The motor is moved in the chosen direction provided the random number is smaller than the corresponding rate. This protocol ensures relaxation to equilibrium in the absence of chemical or mechanics driving forces. Time in the simulations is measured by the number of attempted moves.

\section{APPENDIX B: EVOLUTION OPERATOR}

Here we describe the construction of the evolution operator corresponding to model Eq. (11). First consider the case when the rates for leaving the track are set to zero. To do this we write the Master equation describing the time evolution as

$$
\partial_{t}|P(t)\rangle=\mathcal{M}|P(t)\rangle
$$

where $|P(t)\rangle$ is a vector with components $p_{n}(t)$, the probability of being at site $n$ at time $t[31]$. $\mathcal{M}$ is the evolution operator of the model with non-zero components given by

$$
\begin{aligned}
\mathcal{M}_{i, i+1} & =w_{b}^{\leftarrow} \quad \mathcal{M}_{i, i-1}=w_{b} \rightarrow \text { for } i \text { even } \\
\mathcal{M}_{i, i+1} & =w_{a}^{\leftarrow} \quad \mathcal{M}_{i, i-1}=w_{a} \rightarrow \text { for } i \text { odd } \\
\mathcal{M}_{i, i} & =-\mathcal{M}_{i-1, i}-\mathcal{M}_{i+1, i} .
\end{aligned}
$$




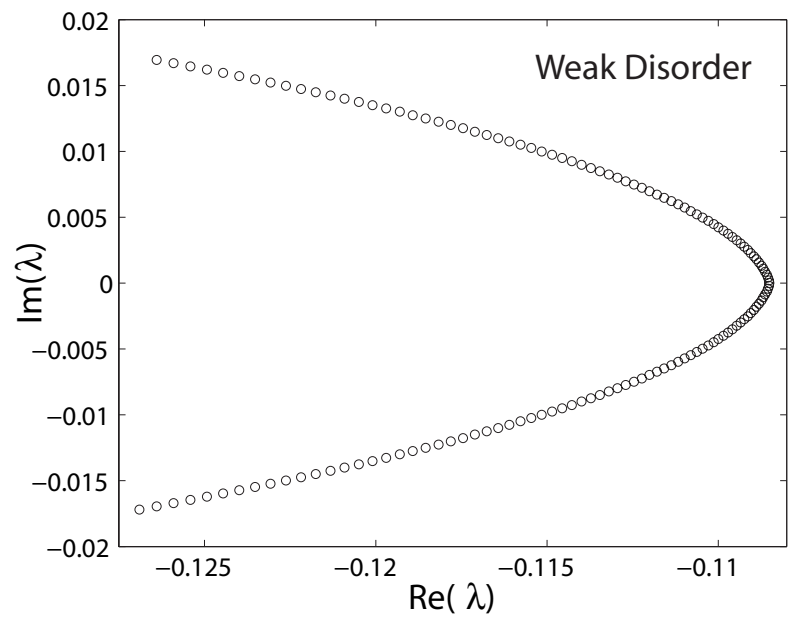

FIG. 20: The eigenvalue spectrum for the random energy model with rates for hopping off the track as specified in the text. Here an example of the weak disorder regime spectrum is shown. Note that the right most part of the $\operatorname{Re}(\lambda)$ axis is displaced slightly below zero. Shown are the 140 eigenvalues with the lowest value of $|\lambda|$ and the system size is $N=4500$.

The last relation ensures conservation of probability. For a heterogeneous model the rates corresponding to a given type of monomer $\mathcal{M}_{i, i+1}, \mathcal{M}_{i+1, i}, \mathcal{M}_{i+2, i+1}, \mathcal{M}_{i+1, i+2}$ with $i$ even are chosen at random. The diagonal terms are then automatically given by Eq. B2 To study the eigenvalue spectrum periodic boundary conditions are imposed. Finally, the hopping off rate from site $i, h_{i}$ (given by $w_{\text {off }}^{a}$ or $w_{\text {off }}^{b}$ depending on wether $i$ is even or odd) is added through $\mathcal{M}_{i, i} \rightarrow \mathcal{M}_{i, i}-h_{i}$. The spectra presented in this paper were calculated using MatLab.

\section{APPENDIX C: RANDOM ENERGY MODEL}

In this Appendix we discuss the spectrum of the random energy version of the model, described by Eq. 1 which also allows the energy at even sites to vary. The rates are slightly modified so that they to satisfy local detailed balance with respect to these energies. An example of the relevance of random energy models to biological system can be found in [32], which studies the diffusion of regulatory proteins along DNA. Another realization (although there is no analogy of "falling off") is translocation of ssDNA through a pore with identical environments on the trans and cis sides [11]. As stated in the text, the long time large wavelength properties of this model are very similar to those of a model studied in the context of vortex physics [19]. We use model (1) with $T=1, \Delta \mu=0, T=1$ and use the parameters $\left\{p_{1}\right\}=\left\{\alpha_{1}, \alpha_{1}^{\prime}, \omega_{1}, \omega_{1}^{\prime}, \varepsilon_{1}, \varepsilon_{1}^{\prime}\right\}=\{6,1,1,6,0,1.5\}$ and $\left\{p_{2}\right\}=\left\{\alpha_{2}, \alpha_{2}^{\prime}, \omega_{2}, \omega_{2}^{\prime}, \varepsilon_{2}, \varepsilon_{2}^{\prime}\right\}=\{1.2,1,1,1.2,0.5,0.8\}$. Here $\varepsilon_{i}$, $\varepsilon_{i}^{\prime}$ denotes that energy at even and odd sites respectively.

In Fig. [20] and 21] the spectra of the evolution operator of the model are shown for weak $\left(w_{\mathrm{off}}^{a}=0.1\right.$ and $w_{\mathrm{off}}^{b}=0.1$ $\left(w_{\mathrm{off}}^{a}=0.12\right.$ and $\left.w_{\mathrm{off}}^{b}=0.12\right)$ for the parameter set $\left.\left\{p_{1}\right\}\left(\left\{p_{2}\right\}\right)\right)$ and strong disorder $\left(w_{\mathrm{off}}^{a}=0.1\right.$ and $w_{\mathrm{off}}^{b}=0.1$ $\left(w_{\text {off }}^{a}=0.4\right.$ and $\left.w_{\text {off }}^{b}=0.4\right)$ for the set $\left\{p_{1}\right\}\left(\left\{p_{2}\right\}\right)$ in the detachment rates. As can be seen from the figures strong enough disorder in the hopping off rates, as before, causes eigenvalue with small $|\lambda|$ to be real and therefore associated with localized eigenfunctions [19].

[1] C. Bustamante, Z. Bryant and S. B. Smith, Nature, 42, 423 (2003).

[2] K. Visscher, M. J. Schnitzer and S. M. Block, Nature, 400, 184 (1999).

[3] R. J. Davenport, G. J. L. Wuite, R. Landick and C. Bustamante, Science 287, 2497 (2000).

[4] M. D. Wang, M. J. Schnitzer, H. Yin, R. Landick, J. Gelles and S. M. Block, Science, 282, 902 (1998).

[5] G. J. L. Wuite, S. B. Smith, M. Young, D. Keller and C. Bustamante, Nature. 404, 103 (2000).

[6] T. Ha, I. Rasnik, W. Cheng, H. P. Babcock, G. H. Gauss, T. M. Lohman and S. Chu, Nature, 419, 638 (2002).

[7] T. T. Perkins, R. V. Dalal, P. G. Mitsis and S. M. Block, Science, 301, 1914 (2003).

[8] Ribosomal translation of messenger RNA into a protein sequence provides a similar, albeit more complex, example of a "motor" on a heterogeneous track.

[9] A. Yildiz, J. N. Forkey, S. A. McKinney, T. Ha, Y. E. Goldman and P. R. Selvin, Science, 300, 2061 (2003).

[10] T. Harms amd R. Lipowsky, Phys. Rev. Lett., 79, 2895 (1997). 


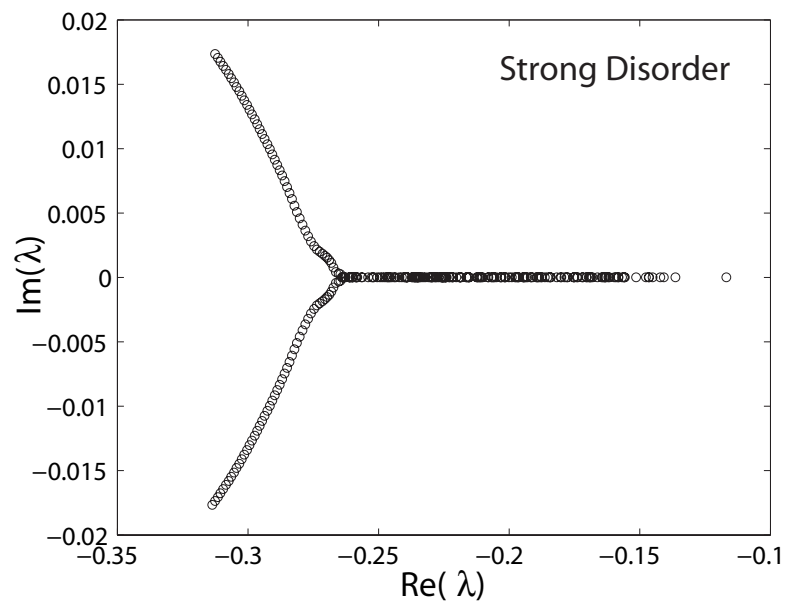

FIG. 21: The eigenvalue spectrum for the random energy model with rates for hopping off the track as specified in the text. Here an example of the strong disorder regime spectrum is shown. Note that the right most part of the Re( $\lambda)$ axis is displaced slightly below zero. Shown are the 300 eigenvalues with the lowest value of $|\lambda|$ and the system size is $N=4500$.

[11] Y. Kafri, D. K. Lubensky and D. R. Nelson, Biophys. J., 86, 3373 (2004).

[12] J. P. Bouchaud, A. Comtet, A. Georges and P. Le Doussal, Ann. Phys. (N. Y.), 201, 285 (1990).

[13] S. Leibler and D. A. Huse, J. Cell. Biol., 121, 1357 (1993).

[14] A. Ajdari, Europhys. Lett., 31, 69 (1995).

[15] R. Lipowsky, S. Klumpp and T. M. Nieuwenhuizen, Phys. Rev. Lett., 87, 108101 (2001).

[16] A. Parmeggiani, T. Franosch, E. Frey, Phys. Rev. Lett., 90, 086601 (2003).

[17] A. Parmeggiani, F. Julicher, L. Peliti and J. Prost, Europhys. Lett., 56, 603 (2001).

[18] N. Hatano and D. R. Nelson, Phys. Rev. Lett., 77, 570 (1996).

[19] N. Hatano and D. R. Nelson, Phys. Rev. B, 56, 8651 (1997); 58, 8384 (1998).

[20] M. E. Fisher and A. B. Kolomeisky, Proc. Natl. Acad. Sci. USA, 96, 6597 (1999).

[21] A. B. Kolomeisky and M. E. Fisher, Biophys. J., 84, 1642 (2003).

[22] J. Prost, J.-F. Chauwin, L. Peliti and A. Ajdari, 72, 2652 (1994).

[23] F. Jülicher, A. Ajdari and J. Prost, Rev. Mod. Phys., 69, 1269 (1997).

[24] Note that the same energy landscape could be obtained by decimating over even sites and allowing $\Delta \varepsilon$ to vary.

[25] B. Derrida, J. Stat. Phys., 31, 433 (1983).

[26] D. R. Nelson and N. M. Shnerb, Phys. Rev. E, 58, 1383 (1998).

[27] K. A. Dahmen,D. R. Nelson, N. M. Shnerb, J. Math. Biol. 41, 1 (2000).

[28] N. M. Shnerb and D. R. Nelson, Phys. Rev. Lett., 80, 5172 (1998); K. A. Dahmen, D. R. Nelson and N. Shnerb, in The Statisitcal Mechanics of Biocomplexity, edited by D. Reguera et. al., (Springer, Berlin, 1999) p. 144.

[29] L. D. Landau and E. M. Lifshitz, Quantum Mechanics, 3rd edition (Pergamon, New York, 1977).

[30] P. Grassberger and I. Procaccia, Phys. Rev. A, 26, 3686 (1982).

[31] N. G. Van Kampen, Stochastic Processes in Physics and Chemistry, Elsevier, Amsterdam (1992).

[32] M. Slutsky, M. Kardar and L. A. Mirny, q-bio/0310008 